\title{
Mathematical Morphology based Retinal Image Blood Vessels Segmentation
}

\author{
R. Adalarasan,R. Malathi
}

\begin{abstract}
It is necessary to verify the state of blood vessel network in the retina for diagnosing various issues associated with eyes. In this research paper, an involuntary retinal vessel segmentation using mathematical morphology is proposed. The contrast of the retinal images is enhanced by contrast limited adaptive histogram equalisation technique. Ten blood vessels of the enhanced retinal image are detected using morphological processing. The hysteresis thresholding is applied on the blood vessels detected image to remove the unwanted back ground detail. Finally the properly segmented binary image of the retinal vessel is obtained using post processing process. Results of the presented method are verified by using most widely used for benchmarking retinal image databases such as, Child Heart and Health Study in England (CHASE_DB1) and Digital Retinal Images for Vessel Extraction (DRIVE) database by computing the evaluation metrics such as sensitivity, specificity, accuracy and precision. The better evaluation metrics achieved for the DRIVE dataset are $0.7493,0.9687,0.9524$ and 0.6590 , and the worst values are $0.6621,0.9411,0.9137$ and 0.5491 . The best evaluation metrics values for the CHASE_DB1 dataset are 0.5058, 0.8947, 0.9382 and 0.8856 , and the worst values are $0.5639,0.9581$, 0.9137 and 0.7110 . The investigational results show that the suggested approach provides the excellent accuracy in comparison with other approaches.
\end{abstract}

Keywords: Blood Vessel segmentation, Morphological processing, Retinal images.

\section{INTRODUCTION}

Nowadays, the retinal fundus imaging becomes popular in research community for monitoring several eye disorders. It plays a vital role in providing assistance to ophthalmologists and cardiologists. However, the quantitative evaluation requires appropriate segmentation of the vascular tree. The involuntary and accurate segmentation is important because labour-intensive segmentation of blood vessels in retinal images is both time intense and error-prone process for skilled physicians. [28-30].

Numerous numbers of papers published about the automatic blood vessels segmentation and still the segmentation accuracy results are not in the acceptable level.

Revised Manuscript Received on October 30, 2019.

* Correspondence Author

R.Adalarasan, EIE Department, Annamalai University, Chidambaram, Tamil Nadu, India. Email:adalarasanece@gmail.com

R.Malathi*, EIE Department, Annamalai University, Chidambaram, Tamil Nadu, India. Email: vsmalu@gmail.com

(c) The Authors. Published by Blue Eyes Intelligence Engineering and Sciences Publication (BEIESP). This is an open access article under the CC-BY-NC-ND license http://creativecommons.org/licenses/by-nc-nd/4.0/
In this research paper, an involuntary retinal vessel segmentation using mathematical morphology is proposed. The contrast of the retinal images quality is improved by contrast limited adaptive histogram equalisation technique. Then blood vessels of the enhanced retinal image are detected using morphological processing. The hysteresis thresholding is applied on the blood vessels detected image to remove the unwanted back ground detail. Finally the properly segmented binary image of the retinal vessel is obtained using post processing process.

The rest of the research paper is structured as follows: Section 2 deals with the survey of the blood vessels segmentation algorithms present in the literature followed by summary of existing blood vessels segmentation techniques. Section 3 gives detail description of the presented retinal blood vessels segmentation algorithm. Detail about the benchmark retinal image datasets used in our experimentation is given in Section 4. Section 5 demonstrates the performance in terms of the evaluation metrics used to show the efficacy of the presented algorithm. The experimental analysis of the proposed framework is given in Section6. In Section 7 deals with concluding remarks which is then followed by future challenges.

\section{LITERATURE REVIEW}

In this Section, the numerous existing retinal blood vessels segmentation algorithms are discussed along with its drawbacks.

Marín et al. [1] has introduced a new blood vessel segmentation algorithm based on supervised neural network. The retinal images are pre-processed by extracting a 7-D feature vector and these features are given as input to a neural network. Post processing technique is applied to fill the pixel gaps in the detected blood vessels which intern eliminate falsely-detected isolated vessel pixels. Although method is very simple, it achieves high accuracy under different illumination conditions proving its robustness and simplicity in systems for computerized detection of eye diseases. Gegundez-Arias et al.[2] have proposed a new evaluation metric, based on the evaluation of measurable features relating vasculature. Precisely, this technique allows vascular structure impost through its characterization. In Salazar-Gonzalez et al. [3], a novel segmentation technique has presented which initially uses graph cut method to segment the vascular structure of the retina and uses blood vessel information to locate the optic disk. Author has employed two different segmentation techniques. 


\section{Mathematical Morphology based Retinal Image Blood Vessels Segmentation}

Experiments results have proved that this segmentation method overcomes the disadvantages in particular the intersecting tissue segmentation.

A new supervised retinal blood vessel segmentation method using deep neural network is presented by Li et al. [4]. The presented technique outperforms other standard techniques in terms of the evaluation metrics. The outcome of cross-training estimation specifies its toughness to the training set. This technique reduces the computational burden of system as it does not require artificially designed features and pre-processing steps.

In Zhang et al. [5]), to attain the proper position with the local structures a new method proposed. The LAD frame is created. The LAD frame provides the multi-scale filtering and enhanced the retinal blood vessels. The presented technique outperforms other standard techniques. Morphology based global thresholding is proposed in Jiang et al.[6] to extract the retinal structures. This technique not only provides greater accuracy and superior robustness, but also decreases the computational burden of system and reduces the execution time.

Eladawi et al. [7] introduced involuntary retinal blood vessels segmentation system using Optical Coherence Tomography Angiography (OCTA) images. A pre-processing and segmentation of blood vessels is done through the GGMRF model and MGRF model. Jiang et al. [8] have suggested a overseen way to segment retinal blood vessel using convolutional network with transfer learning. The suggested technique is very simple and shown the excellent performance over the other conventional retinal blood vessel segmentation algorithms.

Girard, Kavalec, and Cheriet [9] have proposed a semantic segmentation method that uses deep-learning techniques to segment and classify the retinal blood vessels into veins and arteries. This method is fast and definitely scalable to any size of fundus image. It also employs a novel global arterio-venous ratio (AVR) measure to detect significant changes in diabetic retinopathy (DR) cases. Chudzik et al. [10] proposed blood vessels segmentation approach and reported the performance of their method with the DRIVE and STARE databases. They achieved AUC of 0.964 on the DRIVE database and an AUC of 0.983 on the STARE database. Since segmentation results not shown in the paper, very difficult to predict the robustness of the technique. Another method proposed by Hajabdollahi et al [11], and achieved excellent performances with an AUC of 0.97 and an accuracy of 0.961 on the STARE database. Soomro et al [12] verified their method on the two benchmarking databases, and the performance of the method is highly comparable to that of other existing approaches. Tan et al [13] suggested a 7-layer CNN based method to segmenting the retinal blood vessels. Their accuracy is low. The summary of the existing algorithms on the topic of our research is given in Table-I .

\section{PROPOSED BLOOD VESSELS SEGMENTATION ALGORITHM}

The detail explanation of the proposed algorithm is given below in the form of steps. It is also expressed in the form of block diagram in Fig.1.

\section{Step1: Retinal image pre-processing}

To enhance the retinal image, CLAHE technique is applied on the complemented green channel of the input retinal image.
Step2: Vessel detection using morphological operations The retinal vessel segmentation is done using morphological operations. It applies the structuring element to the image and output the image of same size. The morphological dilation and open operation is performed on enhanced retinal image. Opening is a process that does morphological erosion followed by dilation. Output image from dilation operation subtracted from Output image from opening operation. Then closing operation is performed on difference image.

Step3: Retinal image segmentation using hysteresis thresholding

The hysteresis threshold is applied on the extracted retinal vessels. A hysteresis thresholding uses two thresholds. After applying the threshold the back ground is eliminated and the properly segmented binary image of the retinal vessel is obtained.

\section{Step4: Post processing of segmented retinal image}

Post processing of segmented retinal image is done to segment the retinal image by eliminating the small objects and filling the small holes.

\section{Step5: Performance evaluation}

In the final stage, performance of the presented system is evaluated in terms of the evaluation metrics such as Accuracy, Specificity, Sensitivity and precision by comparing obtained segmentation results with the manual segmented retinal images.

\section{RETINAL IMAGE DATABASES}

Digital Retinal Images for Vessel Extraction (DRIVE) [14] and CHASE_DB1 [15] are two most widely used databases to analysis the retinal images. DRIVE database has 40 images, 40 images are split into two groups namely training and testing images. A spatial resolution of each image is $768 \times 584$ pixels. Each image has its own mask images and manually segmented images. CHASE_DB1 dataset comprises of 28 colour fundus images captured with a Nidek NM 200D fundus camera from patients. A number of pixels of each image are $1280 \times 960$. Along with this manual segmentation results of vessel tree are provided.

\section{Evaluation Metrics}

The performance of the existing retinal image vessels segmentation was assessed by measuring the four metrics such as accuracy, True positive rate (sensitivity), specificity and precision. These metrics are expressed as follows:

1) Accuracy metric can be expressed as

\section{Accuracy $(\mathrm{AC})=\frac{\text { sum of correctly identified vessels and non }- \text { vessels }}{\text { total number of pixels }}$}

2) Sensitivity (True Positive Rate), replicates the capability of the algorithm to identify the vessel' pixels. It is expressed as

$$
\text { Sensitivity }(\mathrm{Se})=\frac{\text { correctly identified vessels }}{\text { total number of vessels }}
$$

3) Specificity, ability to identify non-vessel pixels. It can be expressed as

$$
\text { Specificity }(S p)=\frac{t n}{t n+f p}
$$


4) Precision measure can be expressed as

$$
\text { Precision }=\frac{t p}{t p+f p}
$$

Where $t p=$ true positive,

$t n=$ true negative,

$f p=$ false positive

$f n=$ false negative. Accuracy is the foremost measure that provides the overall classification performance of the vessels pixels.

\section{EXPERIMENTAL ANALYSIS}

All the experiments are conducted using MATLAB2018a on PC with 128GB RAM and 2.9GHz CPU. The performance of the presented blood vessels segmentation method is assessed over two benchmark datasets i.e. DRIVE and CHASE_DB1. To assess the performance of the presented retinal image blood vessels segmentation algorithm, we calculate the specificity, sensitivity, accuracy and precision for the benchmark datasets such as DRIVE and CHASE_DB1. We also show the segmented blood vessels of the DRIVE retinal image datasets in Fig.2 and CHASE_DB1 retinal image datasets in Fig.3. For each retinal image in the datasets presented morphological based segmentation is applied and the evaluation metrics are measured by taking the ground truth segmentation results. These metric values are given in Table-II. Table-III presents average results of the performance metrics for the datasets used for evaluation. The better value of the evaluation metrics for the DRIVE dataset are $0.7493,0.9687,0.9524$ and 0.6590 , and the worst values of are $0.6621,0.9411,0.9137$ and 0.5491 . The best evaluation metrics for the CHASE_DB1 dataset are 0.5058, $0.8947,0.9382$ and 0.8856 , and the worst values are 0.5639 , $0.9581,0.9137$ and 0.7110 .

The performance results of the proposed segmentation algorithm are related to those of the recent retinal image blood vessels segmentation algorithms such as Zhang etal.[5] 2016, Jiang et al.[8] 2018, Salazar-Gonzalez et al.[3] 2014 \& Jiang et al.[6] 2017 in Table 3 for DRIVE and CHASE_DB1 datasets. The proposed segmentation algorithm attains better than the existing recent segmentation algorithms in terms of the evaluation metrics equally for both the benchmark retinal image datasets. It is shown in Fig.4 \& Fig.5.

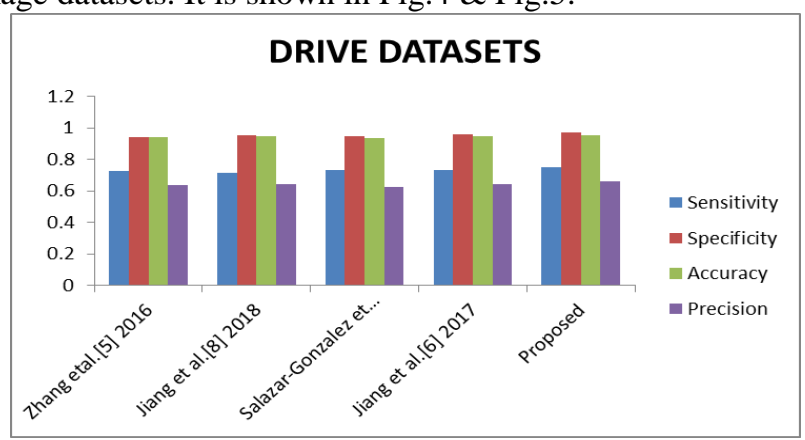

Fig.4 Segmentation results comparison for DRIVE datasets

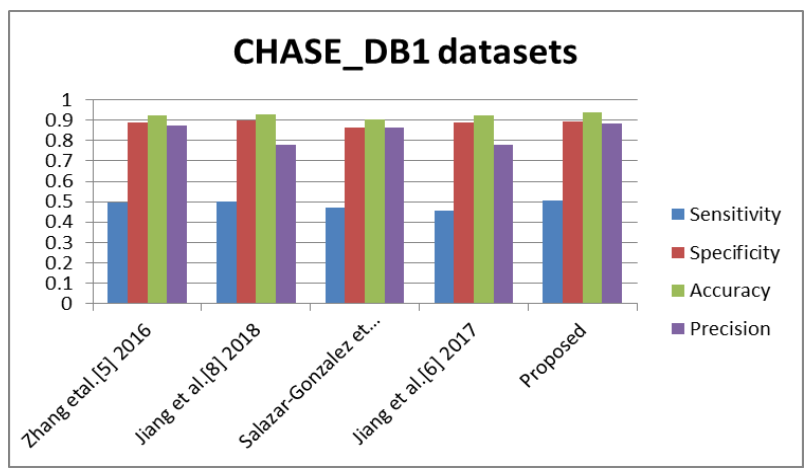

Fig.5 Segmentation results comparison for CHASE_DB1 datasets

\section{Conclusion}

A novel retinal image blood vessels segmentation algorithm using mathematical morphological processing is proposed. The performance of the proposed blood vessels segmentation algorithm is validated against the two benchmark datasets namely DRIVE and CHASE_DB1 in terms of the segmentation evaluation metrics such Sensitivity, Specificity, Accuracy and Precision. It attains the best values of the evaluation metrics for the DRIVE dataset are 0.7493, 0.9687, 0.9524 and 0.6590 , respectively and the worst values are $0.6621,0.9411,0.9137$ and 0.5491 , respectively. The best value of accuracy, precision, sensitivity and specificity for the CHASE_DB1 dataset are 0.5058, 0.8947, 0.9382 and 0.8856, respectively and the worst values are $0.5639,0.9581,0.9137$ and 0.7110 , respectively. Experimentation results prove that the performance of the proposed is superior to the state of the art algorithms in terms of the segmentation evaluation metrics. From the segmented blood vessels retinal images finding various disorders in human eye is the challenging task need to work in future. 
Mathematical Morphology based Retinal Image Blood Vessels Segmentation

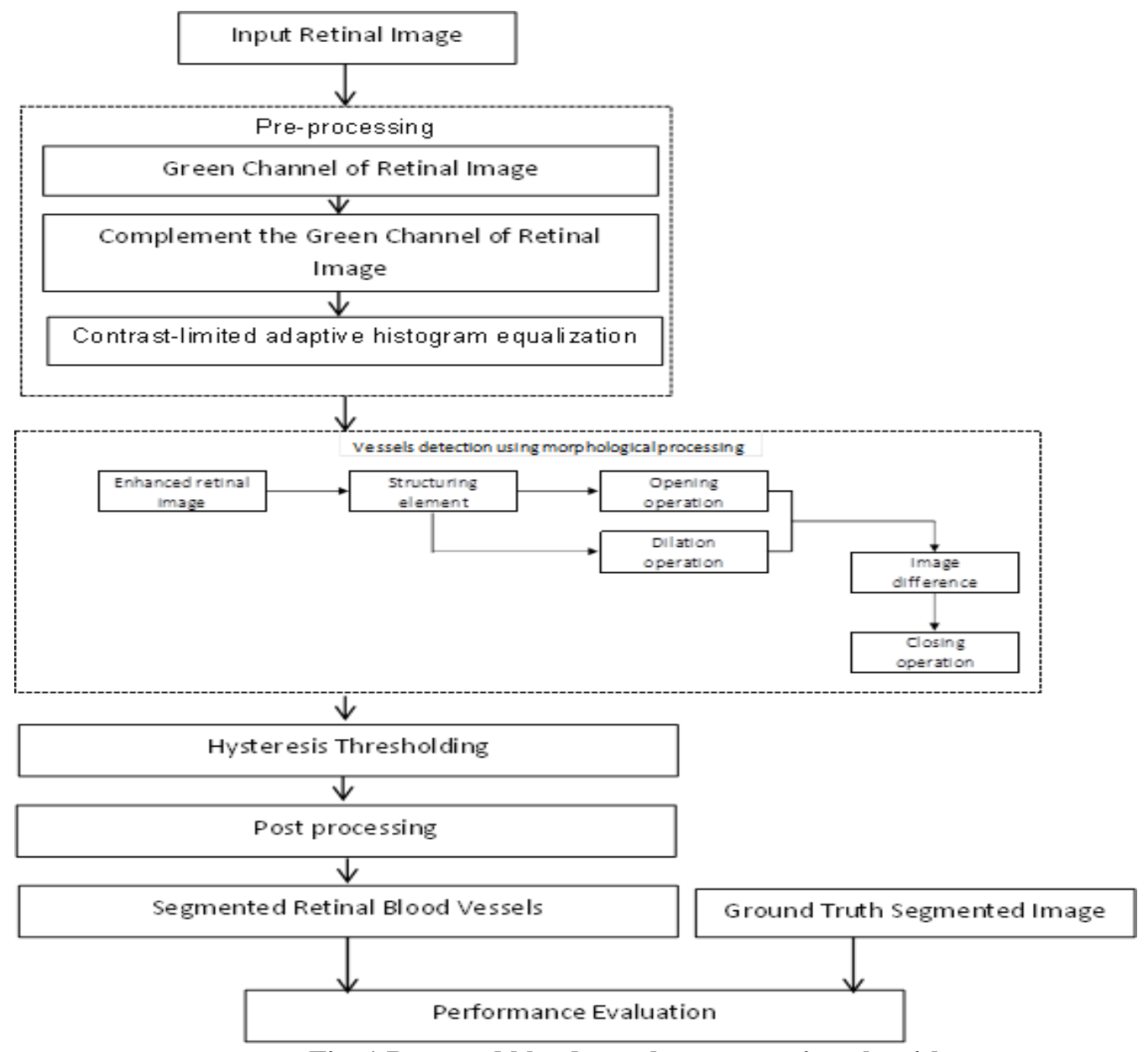

Fig. 1.Proposed blood vessels segmentation algorithm

\begin{tabular}{|l|l|l|l|}
\hline Input image & $\begin{array}{l}\text { Proposed segmented } \\
\text { image }\end{array}$ & $\begin{array}{l}\text { Reference Segmented } \\
\text { image }\end{array}$ \\
\hline & &
\end{tabular}

Published By:

Blue Eyes Intelligence Engineering \& Sciences Publication 


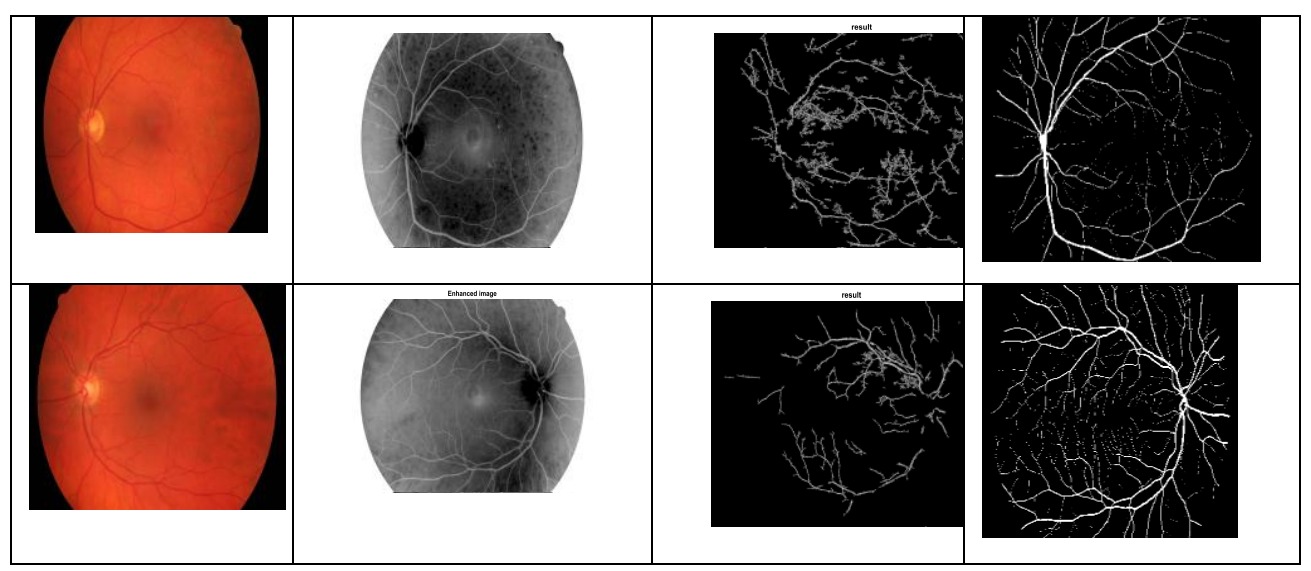

Fig.2 .Segmentation results for DRIVE datasets

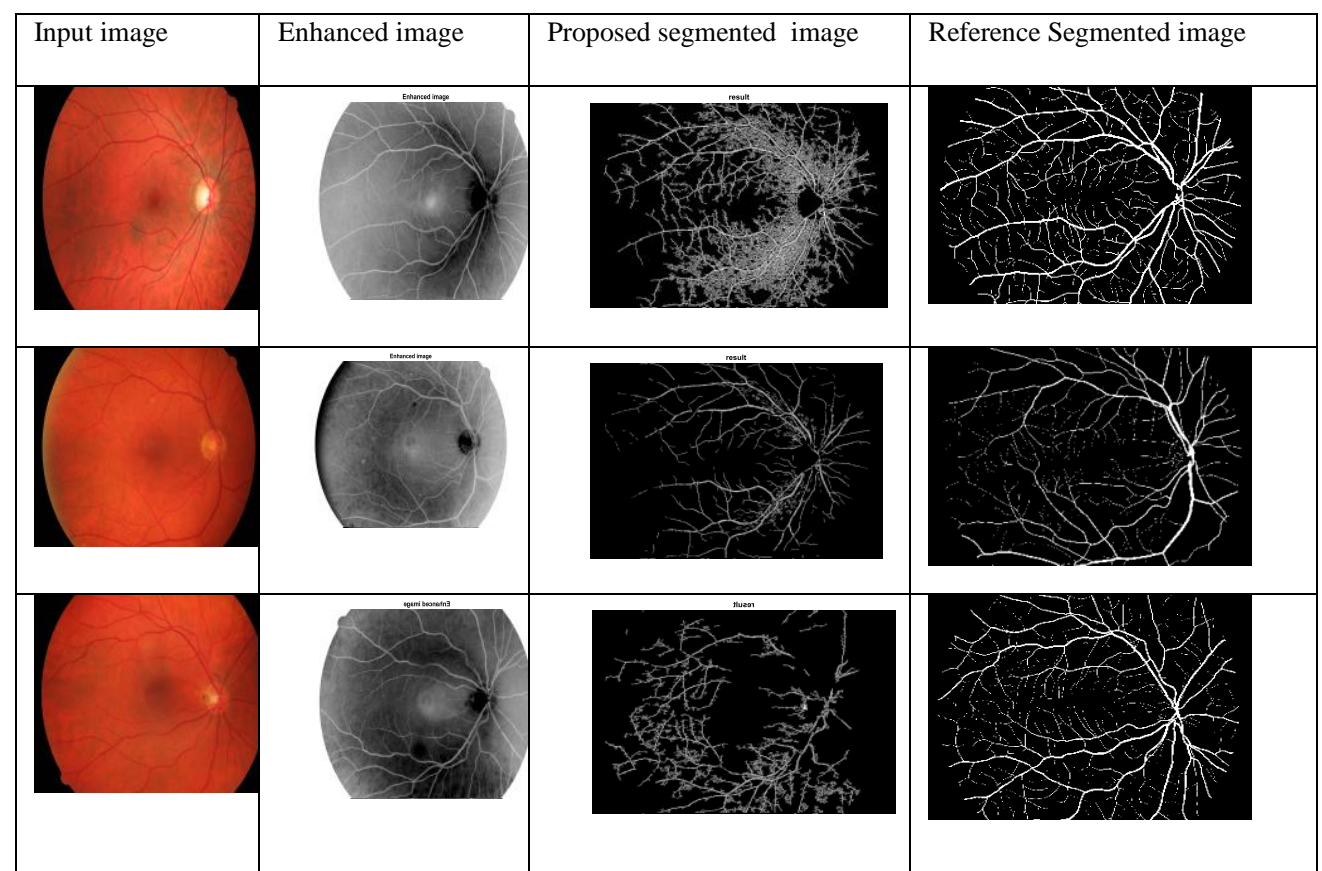

Fig.3 .Segmentation results for CHASE_DB1 datasets

Table-I: Summary of the existing techniques

\begin{tabular}{|c|c|c|c|}
\hline Method, year & Techniques used & Performance Metric & Validation data sets \\
\hline Yin et al. [16] 2010 & $\begin{array}{l}\text { Vessel tracing method based } \\
\text { on statistical approach. }\end{array}$ & $\begin{array}{c}\text { True Positive Rate, } \\
\text { False PR Positive Rate }\end{array}$ & DRIVE \\
\hline Budai et al. [17] 2010 & $\begin{array}{l}\text { Gaussian pyramid } \\
\text { multi-scaling. }\end{array}$ & SE, SP, ACC, & STARE, DRIVE \\
\hline Marín et al. [1] 2011 & $\begin{array}{l}\text { Neural network (NN) scheme } \\
\text { for pixel classification }\end{array}$ & SE, SP, ACC, & STARE, DRIVE \\
\hline $\begin{array}{l}\text { Kaur and Sinha [18], } \\
2012\end{array}$ & $\begin{array}{c}\text { Gabor filter used as Filter } \\
\text { Kernel }\end{array}$ & ROC & STARE, DRIVE \\
\hline $\begin{array}{l}\text { Xie, S.\& Nie, H. [26] } \\
2013\end{array}$ & Genetic algorithm+FCM & ROC,ACC & DRIVE, STARE \\
\hline $\begin{array}{l}\text { Odstrcilik et al. [19], } \\
2013\end{array}$ & $\begin{array}{l}\text { Improved Gaussian matched } \\
\text { filter of t-dimensional. }\end{array}$ & SE, SP, ACC, & STARE and DRIVE \\
\hline $\begin{array}{r}\text { Zolfagharnasab et al. } \\
{[20], 2014}\end{array}$ & $\begin{array}{l}\text { Caushy PDF used as Filter } \\
\text { Kernel }\end{array}$ & ACC, FPR & DRIVE \\
\hline $\begin{array}{l}\text { Salazar-Gonzalez et } \\
\text { al.[3] } 2014\end{array}$ & $\begin{array}{c}\text { Markov random field (MRF) + } \\
\text { Graph cut segmentation }\end{array}$ & TPR, FPR, ACC & $\begin{array}{c}\text { DIARETDB1, } \\
\text { DRIVE, and STARE }\end{array}$ \\
\hline Singh et al. [21],2015 & $\begin{array}{c}\text { Entropy thresholding and } \\
\text { Modified Gaussian matched filter }\end{array}$ & SE, SP, ACC, & DRIVE \\
\hline Zhao et al. [22],2015 & Active contour Approach & SE, SP, ACC, & STARE and DRIVE \\
\hline Kumar et al. [23] 2016 & $\begin{array}{l}\text { Laplacian of Gaussian used as } \\
\text { Filter Kernel }\end{array}$ & SE, SP, ACC, & STARE, DRIVE \\
\hline
\end{tabular}


Mathematical Morphology based Retinal Image Blood Vessels Segmentation

\begin{tabular}{|c|c|c|c|}
\hline De et al. [24] 2016 & Graph theory & GFPR & STARE and DRIVE \\
\hline Maninis et al. [25] 2016 & $\begin{array}{c}\text { Deep Convolutional Artificial } \\
\text { Neural Networks }\end{array}$ & ROC & STARE, DRIVE \\
\hline Li et al. [4] 2016 & $\begin{array}{c}\text { cross-modality learning by } \\
\text { deep neural network }\end{array}$ & ACC, SP, SE, ROC & $\begin{array}{c}\text { DRIVE, STARE, } \\
\text { CHASE_DB1 }\end{array}$ \\
\hline Zhang etal.[5] 2016 & $\begin{array}{c}\text { A locally adaptive and } \\
\text { left-invariant rotating derivative } \\
\text { frame }\end{array}$ & AUC, SP, SE, ACC & $\begin{array}{c}\text { DRIVE, STARE, } \\
\text { CHASE_DB1 }\end{array}$ \\
\hline Eladawi etal.[7] 2017 & $\begin{array}{c}\text { Generalized Gauss-Markov } \\
\text { random field (GGMRF) model }\end{array}$ & AUC, DSC, VVD & mild DR cases \\
\hline Jiang et al. [27] 2017 & $\begin{array}{c}\text { Morphological and Global } \\
\text { thresholding operations }\end{array}$ & Acc, Execution time & STARE, DRIVE \\
\hline Jiang et al.[6] 2017 & $\begin{array}{c}\text { Morphology based global } \\
\text { thresholding }\end{array}$ & SE, SP, ACC & STARE, DRIVE \\
\hline Jiang et al.[8] 2018 & $\begin{array}{c}\text { with transfer learning } \\
\text { concorporated in fully } \\
\text { convolutional network }\end{array}$ & AUC, SP, SE, ACC & STARE, DRIVE, \\
CHASE_DB1 and HRF \\
\hline $\begin{array}{l}\text { Girard, Kavalec, and } \\
\text { Cheriet [9] 2019 }\end{array}$ & $\begin{array}{c}\text { Convolutional neural network } \\
\text { (CNN) }\end{array}$ & ACC, SP, SE, & CT-DRIVE \\
\hline
\end{tabular}

ROC: Receiver Operating Characteristics; ACC: Accuracy; SP: Specificity; SE: Sensitivity;

FPR: False Positive Rate; GFPR: Geometric False Positive Rate

Table-II: Segmentation evaluation metrics

\begin{tabular}{|c|c|c|c|c|c|}
\hline Test Image & Datasets & $\begin{array}{c}\text { Sensitivit } \\
\mathbf{y}\end{array}$ & $\begin{array}{c}\text { Specificit } \\
\mathbf{y}\end{array}$ & $\begin{array}{c}\text { Accurac } \\
\mathbf{y}\end{array}$ & $\begin{array}{c}\text { Precisio } \\
\text { n }\end{array}$ \\
\hline 1 & \multirow{5}{*}{ DRIVE } & 0.7493 & 0.9687 & 0.9523 & 0.6590 \\
\hline 2 & & 0.7068 & 0.9668 & 0.9433 & 0.6788 \\
\hline 3 & & 0.6621 & 0.9411 & 0.9137 & 0.5491 \\
\hline 4 & & 0.2736 & 0.9601 & 0.9251 & 0.2685 \\
\hline 5 & & 0.2241 & 0.9877 & 0.9365 & 0.5661 \\
\hline 6 & \multirow{3}{*}{$\underset{1}{\text { CHASE_DB }}$} & 0.5420 & 0.9905 & 0.9243 & 0.8020 \\
\hline 7 & & 0.5058 & 0.8942 & 0.9382 & 0.8856 \\
\hline 8 & & 0.5639 & 0.9581 & 0.9137 & 0.7110 \\
\hline
\end{tabular}

Table-III: Results Comparison for the DRIVE and CHASE_DB1 databases.

\begin{tabular}{|c|c|c|c|c|c|c|c|c|}
\hline \multirow[b]{2}{*}{ Method } & \multicolumn{4}{|c|}{ DRIVE } & \multicolumn{4}{|c|}{ CHASE_DB1 } \\
\hline & Sensitivity & Specificity & Accuracy & Precision & Sensitivity & Specificity & Accuracy & Precision \\
\hline Zhang etal.[5] 2016 & 0.7289 & 0.9402 & 0.9387 & 0.6389 & 0.4983 & 0.8879 & 0.9261 & 0.8764 \\
\hline Jiang et al.[8] 2018 & 0.7134 & 0.9534 & 0.9456 & 0.6432 & 0.5029 & 0.8975 & 0.9302 & 0.7802 \\
\hline $\begin{array}{c}\text { Salazar-Gonzalez et } \\
\text { al.[3] } 2014\end{array}$ & 0.7295 & 0.9489 & 0.9329 & 0.6267 & 0.4709 & 0.8629 & 0.9029 & 0.8640 \\
\hline Jiang et al.[6] 2017 & 0.7337 & 0.9570 & 0.9484 & 0.6406 & 0.4568 & 0.8890 & 0.9256 & 0.7789 \\
\hline
\end{tabular}

\section{REFERENCES}

1. Marín, Diego, Arturo Aquino, Manuel Emilio Gegúndez-Arias, and José Manuel Bravo.2011. “A New Supervised Method for Blood Vessel Segmentation in Retinal Images by Using Gray-Level and Moment Invariants-Based Features." IEEE Transactions on Medical Imaging 30(1):146-58.

2. Gegundez-Arias, Manuel Emilio, Arturo Aquino, José Manuel Bravo, and Diego Marin.2012. "A Function for Quality Evaluation of Retinal Vessel Segmentations." IEEE Transactions on Medical Imaging 31(2):231-39

3. Salazar-Gonzalez, Ana, Djibril Kaba, Yongmin Li, and Xiaohui Liu. 2014."Segmentation of the Blood Vessels and Optic Disk in Retinal Images." IEEE Journal of Biomedical and Health Informatics 18(6):1874-86.

4. Li, Qiaoliang, Bowei Feng, Linpei Xie, Ping Liang, Huisheng Zhang, and Tianfu Wang. 2016. "A Cross-Modality Learning Approach for Vessel Segmentation in Retinal Images." IEEE Transactions on Medical Imaging 35(1):

5. Zhang, Jiong, Behdad Dashtbozorg, Erik Bekkers, Josien P. W. Pluim, Remco Duits, and Bart M. Ter Haar Romeny. 2016. "Robust Retinal Vessel Segmentation via Locally Adaptive Derivative Frames in Orientation Scores." IEEE Transactions on Medical Imaging 35(12): PP.2631-44.
6. Jiang, Zhexin, Juan Yepez, Sen An, and Seokbum Ko. 2017. "Fast, Accurate and Robust Retinal Vessel Segmentation System." Biocybernetics and Biomedical Engineering 37(3): PP. 412-21.

7. Eladawi, Nabila, Mohammed Elmogy, Omar Helmy, Ahmed Aboelfetouh, Alaa Riad, Harpal Sandhu, Shlomit Schaal, and Ayman El-Baz. 2017. "Automatic Blood Vessels Segmentation Based on Different Retinal Maps from OCTA Scans." Computers in Biology and Medicine 89(April): pp. 150-61.

8. Jiang, Zhexin, Hao Zhang, Yi Wang, and Seok Bum Ko. 2018. "Retinal Blood Vessel Segmentation Using Fully Convolutional Network with Transfer Learning." Computerized Medical Imaging and Graphics 68(April):pp.1-15.

9. Girard, Fantin, Conrad Kavalec, and Farida Cheriet. 2019. "Joint Segmentation and Classification of Retinal Arteries/Veins from Fundus Images." Artificial Intelligence in Medicine 94(February): pp.96-109.

10. Chudzik, B. Al-Dir, F. Caliv, and A. Hunter. 2018, "DISCERN: Generative framework for vessel segmentation using convolutional neural network and visual codebook,' inProc. IEEE 40th Annu. Int. Conf. Eng. Med. Biol.Soc. (EMBC), vol. 1, pp. 5934-5937.

11. M. Hajabdollahi, R. Esfandiarpoor, K. Najarian, N. Karimi, S. Samavi,and S. M. Reza-Soroushmeh, "Low complexity convolutional neural network for vessel segmentation in portable retinal diagnostic devices,' 'in Proc. ICIP, Oct. 2018, pp. 2785-2789. 
12. A. Soomro, O. Hellwich, A. J. Afifi, M. Paul, J. Gao, and L. Zheng, "Strided U-Net model: Retinal vessels segmentation using dice loss,' in Proc. Digit. Image Comput., Techn. Appl. (DICTA), vol. 1, Dec. 2018,pp. 1-8.

13. H. Tan, U. R. Acharya, S. V. Bhandary, K. C. Chua, and S. Sivaprasad,"Segmentation of optic disc, fovea and retinal vasculature using a sin-gle convolutional neural network,'’J. Comput. Sci., vol. 20, pp. 70-79,May 2017

14. J. Staal, M. D. Abramoff, M. Niemeijer, M. A. Viergever, and B. van Ginneken, "Ridge-based vessel segmentation in color images of theretina,' 'IEEE Trans. Med. Imag., vol. 23, no. 4, pp. 501-509, Apr. 2004.

15. J. V. B. Soares, J. J. G. Leandro, R. M. Cesar, Jr., H. F. Jelinek, andJ. Michael Cree, "Retinal vessel segmentation using the 2-D Gabor waveletand supervised classification,'’IEEE Trans. Med. Imag., vol. 25, no. 9,pp. 1214-1222, Sep. 2006.

16. Yin, Y.; Adel, M.; Guillaume, M.; Bourennane, S. A probabilistic based method for tracking vessels in retinal images. In Proceedings of the 2010 IEEE International Conference on Image Processing, Hong Kong, China, 26-29 September 2010; pp. 4081-4084.

17. Budai, A.; Michelson, G.; Hornegger, J. Multiscale Blood Vessel Segmentation in Retinal Fundus Images. In Proceedings of the Bildverarbeitung für die Medizin, Aachen, Germany, 14-16 March 2010; pp. 261-265.

18. Kaur, J.; Sinha, H. Automated detection of retinal blood vessels in diabetic retinopathy using Gabor filter. Int. J. Comput. Sci. Netw. Secur. 2012, 12, 109

19. Odstrcilik, J.; Kolar, R.; Budai, A.; Hornegger, J.; Jan, J.; Gazarek, J.; Kubena, T.; Cernosek, P.; Svoboda, O.; Angelopoulou, E. Retinal vessel segmentation by improved matched filtering: Evaluation on a new high-resolution fundus image database. IET Image Process. 2013, 7, 373-383.

20. Zolfagharnasab, H.; Naghsh-Nilchi, A.R. Cauchy Based Matched Filter for Retinal Vessels Detection. J. Med. Signals Sens. 2014, 4, 1-9.

21. Singh, N.P.; Kumar, R.; Srivastava, R. Local entropy thresholding based fast retinal vessels segmentation by modifying matched filter. In Proceedings of the International Conference on Computing, Communication \& Automation, Noida, India, 15-16 May 2015; pp. 1166-1170.

22. Zhao, Y.; Rada, L.; Chen, K.; Harding, S.P.; Zheng, Y. Automated vessel segmentation using infinite perimeter active contour model with hybrid region information with application to retinal images. IEEE Trans. Med. Imaging 2015, 34, 1797-1807.

23. Kumar, D.; Pramanik, A.; Kar, S.S.; Maity, S.P. Retinal blood vessel segmentation using matched filter and laplacian of gaussian. In Proceedings of the 2016 International Conference on Signal Processing and Communications (SPCOM), Bangalore, India, 12-15 June 2016; pp. 1-5.

24. De, J.; Cheng, L.; Zhang, X.; Lin, F.; Li, H.; Ong, K.H.; Yu, W.; Yu, Y.; Ahmed, S. A graph-theoretical approach for tracing filamentary structures in neuronal and retinal images. IEEE Trans. Med. Imaging 2016, 35, 257-272.

25. Maninis, K.-K.; Pont-Tuset, J.; Arbeláez, P.; Van Gool, L. Deep retinal image understanding. In International Conference on Medical Image Computing and Computer-Assisted Intervention; Springer International Publishing: Berlin/Heidelberg, Germany, 2016; pp. 140-148.

26. Xie, S.; Nie, H. Retinal vascular image segmentation using genetic algorithm Plus FCM clustering. In Proceedings of the 2013 Third International Conference on Intelligent System Design and Engineering Applications (ISDEA), Hong Kong, China, 16-18 January 2013; pp. 1225-1228.

27. Jiang, Z.; Yepez, J.; An, S.; Ko, S. Fast, accurate and robust retinal vessel segmentation system. Biocybern. Biomed. Eng. 2017, 37, $412-421$.

28. Prabukumar, M., Agilandeeswari, L. and Sangaiah, A. K. (2017) An Optimized Breast Cancer Diagnosis System Using a Cuckoo Search Algorithm and Support Vector Machine Classifier, in Hybrid Intelligence for Image Analysis and Understanding (eds S. Bhattacharyya, I. Pan, A. Mukherjee and P. Dutta), John Wiley \& Sons, Ltd, Chichester, UK. doi: 10.1002/9781119242963.ch13

29. Prabukumar, M., Prasenjit, N. and Sangeetha, V. (2013) , Feature extraction method for breast cancer diagnosis in digital mammograms using multi-resolution transformations and SVM-fuzzy logic classifier, Int. J. Computational Vision and Robotics, Vol. 3, No. 4, pp279-292 .

30. Prabukumar M, Agilandeeswari, L. and Ganesan. K, An intelligent lung cancer diagnosis system using cuckoo search optimization and support vector machine classifier, Journal of Ambient Intelligence and Humanized Computing, 10(1), 267-293.

\section{AUTHORS PROFILE}

R. Adalarasan ph.D research scholar in Annamalai University, Chidambaram, Tamil Nadu India. He studied B.E (ECE) in Adhiyamaan college of Engineering, Hosur. He completed Master degree in Arulmigu Meenakshi Amman College of Engineering. He has 15+ years of experience in teaching. He published papers in peer reviewed journal and conferences. His area of interest includes image processing, biomedical and machine learning.

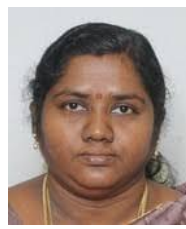

Dr. R. Malathi , Professor \& Director, Centre for Skill Development, Department of Instrumentation Engineering. Words at Annamalai University, Chidambaram, Tamil Nadu India. She published numerous papers in peer reviewed journal and conferences. She is life time member of ISBM, ISCCE, SSI \& SMB. Her area of specializations is computational Bio Engineering and Electro Physiological Modelling. 